\title{
Consumo de tabaco
}

\section{Tabacco consumption}

\author{
Antonio López *, Celsa Perdiz *,**, Encarnación Sueiro *,** \\ * Facultad CC. de la Educación (U. Vigo-Ourense), ${ }^{* *}$ Centro de Orientación Familiar (C.O.F.) (sergas-Ourense)
}

\begin{abstract}
Resumen
Nuestro objetivo es conocer los hábitos tabáquicos en la estapa adolescente. Se trata de un estudio descriptivo y transversal sobre una muestra de 1.128 sujetos a quien se le aplicó una encuesta estructurada para conocer sus conductas relacionadas con el consumo de tabaco. El análisis estadístico se realiza con el SPSS.20 para Windows. Los resultados más relevantes señalan que el $31 \%$ fuma diariamente, el $53.8 \%$ comenzó entre los 13 y 15 años, el $50.4 \%$ consume entre 1 y 5 cigarrillos/día, haciéndolo en el $28,2 \%$ en compañía de otras personas. Señalamos la necesidad de hacer educación para la salud. Palabras clave: tabaco, adolescencia, educación.
\end{abstract}

\section{Abstract}

Our goal is to know the smoking habits in the adolescent stage. This is a descriptive and cross-sectional study of a sample of 1,128 subjects, who was given a structured survey to know their behaviors related to tobacco consumption. Statistical analysis is performed with SPSS.20 for Windows. The most important ones indicate that $31 \%$ smoke daily, $53.8 \%$ started between 13 and 15 years, $50.4 \%$ consume between 1 and 5 cigarettes / day, doing it in $28.2 \%$ with other people. We point out the need to do health education.

Keywords: tabacco, adolescence, education

El tabaco es una de las drogas que máis atención tiene suscitado a los investigadores por los múltiples efectos nocivos que itene sobre la salúd, destacando el cancer de pulmón, la arteriosclerosis y las enfermedades coronarias (Doll e Peto, 1978; USDHHS, 1982, 1989, 1990).

El proceso desde el inicio hasta la dependencia suele durar una media de dos a tres años con inicio en la adolescencia temprana y media (USDHHS, 1988).

E período comprendido entre los 11 y los 15 anos es crítico en el inicio del consumo del tabaco. A los 11 años, en la mayoría de los países, son menos del $20 \%$ quienes probaron el tabaco, pero a los 13 años pasa al $40-50 \%$ y a los 15 años se eleva al 60-70\%. No todos los países participantes tienen los mismos porcentajes de consumo. En los adolescentes españoles se observa un incremento enorme en la experimentación con el tabaco a medida que aumenta la edad en el período comprendido entre los 11 y los 15 años; con porcentajes inferiores al $20 \%$ a los 11 años y cercanos al $60 \%$ a los 15 anos (Mendoza et al., 1994).
En los distintos países, la proporción de los jóvenes que experimentan con el tabaco es maior que la de las jóvenes a edades tempranas (Gabhaine y François, 2000; Mendoza et al., 1994, 1998; USDHHS, 1994), pero a los 15 años esa diferencia desaparece $\mathrm{y}$, a partir de esa edad, las chicas presentan una proporción de experimentación igual o mayor que los chicos. Los datos permiten identificar un patrón geográfico de consumo. No en todos los países la proporción de consumo es igual por sexo, en el caso de España y otros países occidentales, las jóvenes fuman más que los jóvenes (Mendoza et al., 1994, 1998). El INJUVE (2008, 2013) confirma igualmente estos datos manteniéndose esa tendendencia en los dos últimos informes publicados. En este mismo documento, y comparando las dos últimas investigaciones publicadas, se confirma el descenso en el uso de este tóxico.

Los adolescentes de menor estatus socioeconómico presentan mayor tasa de

experimentación con el tabaco (Hendry et al., 1993). Entre los adolescentes españoles también se observa esta relación, pero unicamente en el grupo de chicos (Mendoza et al., 1994).

Nuestra investigación pretende conocer las características del consumo de tabaco en los adolescentes de nuestro medio.

\section{Método}

\section{Objetivos}

- Conocer y comprender mejor al ser humano en su etapa de desarrollo adolescente a traves de los estilos de vida que repercuten en su bienestar .

- Profundizar en el conocimiento de las conductas de consumo de tabaco de 1@s adolescentes escolarizad@s en nuestro medio social.

- Adoptar medidas socioeducativas que repercutan en la prevencion del consumo de tabaco de 1@s adolescentes con quienes trabajamos.

El primer nivel de título irá centrado, en negrita y con únicamente la primera letra en mayúscula. El espaciado anterior será de 10 puntos y el posterior de 3 . La fuente será la misma que para el resto del texto. Se tendrá la precaución de no dejar un título sin texto debajo al final de una página o columna. Los títulos de primer nivel serán: Método, Resultados, Discusión y Referencias. 


\section{Instrumento: Cuestionario estruturado de} elaboración propia

Incluye los siguientes contenidos:

-Variables sociodemográficas: edad y sexo, estado civil, estudios cursados, centro de estudios, lugar de residencia y clase social.

-Variables ideológicas: opción política, religión y práctica religiosa.

-Variables relativas al consumo de tabaco: frecuencia en el consumo, tipo de tabaco, edad de inicio, compañía para el consumo, lugar de consumo, momento del consumo, cantidad de consumo y preocupación sobre las consecuencias del consumo de tabacio sobre la salud.Método, Resultados, Discusión y Referencias.

\section{Muestra}

La investigación se realizó sobre una muestra de 1128 sujetos de edades comprendidas entre los 16 y los 19 años, de ambos sexos, escolarizados en centros públicos y privados, del medio rural y urbano de toda la comunidad gallega. Para la obtención de los datos se recabó colaboración voluntaria en la participación del estudio y para cumplimentación del instrumento de medida.

La edad media es de 17,16 años, siendo la desviación típica de 1,04 años. El 52,7\% son mujeres.

En cuanto a su Lugar de Residencia, el 50,6\%, residen en el medio urbano, el $20,4 \%$ en el Semiurbano y el $29 \%$ en el rural. El 34,5\% de los sujetos son de Lugo, el $25,3 \%$ son de La Coruña, el 21,2\% son de Ourense y el $19,1 \%$ son de Pontevedra.

Por lo que hace referencia a su Clase Social, el 71,2\% se incluyen en la clase social Media, el $22 \%$ en la Media Alta y el 6,8\% en la Media Baja.
Con respecto a sus Estudios, el 60,5\% realiza Bachillerato, el 22,5\% Ciclo Formativo de Grado Medio/Garantía Social y el $16,9 \%$ Ciclo Formativo de Grado Superior/Diversificación Curricular, en Centros Públicos el 60,3\% y Privados/Concertados el 39,7\%.

Con respecto a la Identificación Religiosa, el 73,7\% se declaran católicos o de otras religiones y el 26,3\% de ninguna y con respecto a la Práctica, el $51,3 \%$ dice que alguna vez, el $32 \%$ que nunca y el $16,7 \%$ que siempre o casi siempre.

Y, por lo que se refiere a su Identificación Política, el $52,2 \%$ no se identifica políticamente, el $18,4 \%$ se identifica con el BNG, el 15,5\% con el PP, el 9,8\% con el PSOE y el $5,1 \%$ se identifica con otros partidos políticos.

\section{Análisis estadístico}

El proceso de cálculo se realizó empleando el programa de análisis estadístico SPSS. 20 para Windows. Los datos obtenidos fueron sometidos a un análisis de media y desviación típica, para la variable cuantitativa y de frecuencias y porcentajes de cada una de las variables culitativas.

\section{Diseño}

El estudio es, por una parte, de tipo transversal, en cuanto trata de conocer en una población determinada (adolescentes de 16 a 19 años) y descriptivo, en relación con las conductas de consumo de tabaco en el momento del estudio.

\section{Resultados}

En la siguiente tabla mostramos los resultados obtenidos, relacionados con el consumo de tabaco: 
Tabla 1.

Frecuencia de consumo, edad de inicio, cantidad, marca, compañía para el consumo, lugar, momento del dia y preocupación por las consecuencias del consumo tabaco sobre la salúd (CE)

\begin{tabular}{|c|c|c|}
\hline FRECUENCIA & FRECUENCIA & PORCENTAJE \\
\hline No, nunca & 565 & 50.1 \\
\hline Ocasionalmente & 162 & 14.4 \\
\hline los fines de semana & 48 & 4.3 \\
\hline Diariamente & 353 & 31.3 \\
\hline TOTAL & 1128 & 100 \\
\hline \multicolumn{3}{|l|}{ EDAD DE INICIO } \\
\hline Antes de los 12 años & 79 & 14.0 \\
\hline De $\operatorname{los} 13$ a los 15 años & 303 & 53.8 \\
\hline Dos 16 a los 18 años & 168 & 29.8 \\
\hline Con 19 o mas años & 13 & 2.3 \\
\hline TOTAL & 563 & 100 \\
\hline \multicolumn{3}{|l|}{ CANTIDAD } \\
\hline De 1 a 5 cigarros diarios & 284 & 50.4 \\
\hline De 6 a 10 cigarros diarios & 126 & 22.4 \\
\hline 11 o mái cigarros diarios & 120 & 21.3 \\
\hline Más de una caja diaria & 33 & 5.9 \\
\hline TOTAL & 563 & 100 \\
\hline \multicolumn{3}{|l|}{ MARCA } \\
\hline Chester & 163 & 29.0 \\
\hline Malboro & 142 & 25.2 \\
\hline LM & 83 & 14.7 \\
\hline Camel & 61 & 10.8 \\
\hline Lucky strike & 90 & 16.0 \\
\hline Winston/Fortuna & 16 & 2.8 \\
\hline Ducados & 8 & 1.4 \\
\hline TOTAL & 563 & 100 \\
\hline \multicolumn{3}{|l|}{ COMPAÑÍA } \\
\hline Sólo/ & 41 & 7.3 \\
\hline En compañía da pareja amistades y familia & 159 & 28.2 \\
\hline TOTAL & 563 & 100 \\
\hline \multicolumn{3}{|l|}{$\begin{array}{ll}\text { LUGAR } \\
\end{array}$} \\
\hline En la propia casa y la de amigos & 10 & 1.8 \\
\hline En bares, pubs y discotecas & 192 & 34.1 \\
\hline En todos los lugares anteriores & 361 & 64.1 \\
\hline TOTAL & 563 & 100 \\
\hline \multicolumn{3}{|c|}{ MOMENTO DEL DIA } \\
\hline Por la mañana & 8 & 1.4 \\
\hline Por la tarde & 11 & 2.0 \\
\hline Polr a noche & 92 & 16.3 \\
\hline Por la tarde y por la noche & 81 & 14.4 \\
\hline Por la mañana, tarde y noche & 371 & 65.9 \\
\hline TOTAL & 563 & 100 \\
\hline \multicolumn{3}{|l|}{ PREOCUPACIÓN } \\
\hline Si preocupación & 490 & 87.0 \\
\hline No preocupación & 61 & 10.8 \\
\hline No hay problemas de salúd & 12 & 2.1 \\
\hline TOTAL & 563 & 100 \\
\hline
\end{tabular}

Fuente: Elaboración propia

Tal como se aprecia, el porcentaje de adolescentes que nunca consumieron tabaco $(50.1 \%)$ es muy pareja con los que sí se iniciaron en este tipo de consumo (49.9\%), siendo la edad de inicio mayoritaria entre los 13 y los 15 años (53.8\%), de consumo de 1 a 5 cigarros diários $(50.4 \%)$, marca comercial Chester $(29.0 \%)$, en compañía de pareja, amigos y familia (28.2\%), con consumo en todos los lugares $(64.1 \%)$, por la mañana, tarde y noche $(65.9 \%)$ y con preocupación por las consecuencias que el consumo de tabaco puede ocasionar en la salud (87.0\%).
Por el contrario, quienes representan menores porcentajes son las y los adolescentes que consumen tabaco solo los fines de semana (4.3\%), con edad de inicio en el consumo con 19 o mas años (2.3\%), que consumen mas de una caja diaria (5.9\%), marca comercial Ducados (1.4\%), consumo solitario (7.3\%), en la propia casa y en la de amigos (1.8\%), fuman por la mañana $(1.4 \%)$ y con la creencia de que no hay problemas de salud por el consumo de tabaco (2.1\%). 


\section{Discusión}

Respeto del consumo de tabaco, destacar los resultados de nuestro estudio donde se reflexa que los jóvenes gallegos de 16 a 19 años estudiados en su mayoría nunca consumieron, pero están muy parejos a los que sí iniciaron el hábito siendo tres de cada diez los que consumen diariamente. La edad de inicio se sitúa entre los 13 y los 15 años, en una cantidad de 1 a 5 cigarros diarios, marcas comerciales de tabaco rubio, en compañía de pareja, amigos y familia, en espacios privados y de ocio, en cualquier momento del día y con preocupación por las consecuencias del tabaco sobre la salud.

Nuestros resultados están en la línea de otras investigaciones revisadas. Aburto, Esteban, Quintana, Bilbao y Moraza (2007) encuentran que el $51.8 \%$ de los adolescentes han probado el tabaco, el $29.9 \%$ se ha iniciado con el tabaco entre los 13 y los 15 años. En la misma línea apunta la encuesta estatal ESTUDIES que investiga sobre el consumo de drogas en Estudiantes de Enseñanzas Secundarias 2012-2013 (Ministerio de Sanidad, Política Social e Igualdad, 2013) realizada a chavales de edades entre los 14 y los 18 años que ponen de manifiesto similares edades de inicio y cantidades de consumo pero destacando que aun siendo de las drogas de mayor uso su consumo desciende con respeto a años anteriores.

Paniagua, García, Castellano, Sarrallé y Redondo (2001), destacan que el $80.4 \%$ de los adolescentes consideran el tabaco una droga lo que está en relación con los resultados de nuestro estudio donde se ponen de manifiesto que el $87 \%$ teme las consecuencias del tabaco sobre la salud. Desde la entrada en vigor de la llamada Ley antitabaco Española el 2 de enero del 2011 que prohíbe fumar en calqueir tipo de espacio de uso colectivo que no esté el aire libre (con algunas excepciones) entre otras medidas, la conciencia colectiva general sobre las consecuencias del tabaco sobre la salud ha mudado de manera notoria, mostrándose una tendencia de consumo descendente (Puente, Zabaleta, Pueyo, Saltó, Marsal y Bolívar, 2013) siendo también un factor relevante a inacesibilidade el tabaco (Perez-Milena, MartínezFernández, Redondo-Olmedilla, Álvarez-Nieto, Jímenez-Pulido y Mesa-Gallardo, 2012)

\section{Aportacións para el ámbito socioeducativo}

De los contenidos y datos que arroja nuestra investigación aportamos algunas ideas que a nuestro entender deben ser tenidas en cuenta en los diseños de intervención para el mantenimiento de hábitos de vida saludables y la prevención del hábito de consumo de tabaco. Destacamos las siguientes cuestiones:

La edad en la que se inician los comportamientos de riesgo para la salud es muy precoz en los sujetos de nuestra muestra, por tanto las intervenciones para prevenir deben comenzar también de forma precoz en el medio familiar, evitando trasmitir hábitos nocivos de la conducta de los padres a los hij@s y haciendo educación proactiva de promoción de la vida saludable. Además el medio escolar no puede ser ajeno a esta realidad (desde el inicio mismo de la escolarización de niños y niñas). Planteamos como necesaria la inclusión de la educación para la salud y el bienestar de las personas en el curriculum escolar durante toda la etapa obligatoria. Es imprescindible transmitir la idea de que las personas tenemos un papel autónomo y responsable en la consecución de nuestro estado de salud, que nos afecta de manera individual pero también colectivo. Esta es una decisión política, la vigente y reciente ley educativa (LOMCE, 2013) no considera modificaciones en este sentido.

Padres, comunidad educativa, sistema sanitario y sociedad en general deben tamar conciencia de la importancia de preservar la salud y poner en marcha programas que eviten los primeros contactos y la prevencion primaria del consumo de tabaco.

\section{Referencias}

Aburto, M., Esteban , J.M., Quintana,A., Bilbao F.J., Moraza A. e Capelastegui A. (2007). Prevalencia del consumo de tabaco en adolescentes. Influencia del entorno familiar. An Pediatr (Barc;66(4):357-66

Doll, R., e Peto, R. (1978). Cigarette smoking and bronchial carcinoma: Dose and time relationships among regular smokers and lifelong non-smokers. Journal of Epidemiology and Community Health, 32, 303-313.

Gabhainn, S.N., e François, Y. (2000). Substance use. En C. Currie, K. Hurrelmann, W. Settertobulte, R. Smith \& Todd, J, (Eds.), Health and health behaviour among young people: International report (pp. 97-114). Copenhagen, WHO Regional Office for Europe.

Hendry, L.B., Shucksmith, J., Love, J.G., e Glendinning, A. (1993). Young people's

leisure and lifestyles. London: Routledge.

INJUVE (2008). Informe de la Juventud en España2008. Madrid: Ministerio de Igualdad.

INJUVE (2013). Informe Juventud en España 2012. Madrid: Instituto de la Juventud.

LONCE (2013). Ley orgánica 8/2013, de 9 de diciembre, para la mejora de la calidad educativa. «boe» núm. 295, de 10 de diciembre de 2013, páginas 97858 a 97921

Mendoza, R., Sagrera M. R., e Batista J. M. (1994) Conductas de los escolares españoles relacionadas con la salud (1986-1990). Madrid: C.S.I.C.

Ministerio de Sanidad, Política Social e Igualdad (2014). Informe de la encuesta Estatal sobre uso de drogas en estudiantes de enseñanzas secundarias (ESTUDES) 2012-2013. Madrid: Ministerio de Sanidad, Política Social e Igualdad.

Paniagua, S., García, G., Castellano,R., Sarrallé S. e Redondo , C. (2001). Consumo de tabaco, alcohol y drogas no legales entre adolescentes y relación con los hábitos de vida y el entorno. An Esp Pediatr 55: 121-128

Perez-Milena, A., Martínez-Fernández, M.L., Redondo-Olmedilla, M., Álvarez-Nieto, C., JímenezPulido e Mesa-Gallardo, I. (2012). Motivaciones para el consumo de tabaco entre los adolescentes de 
un instituto urbano. Gaceta Sanitaria, 26(1): 51-57. Título artículo.

Puente, D., Zabaleta, E., Pueyo, M.J., saltó, E., Marsal, J.R. e Bolívar, B. (2013). Prevalencia y factores asociados al consumo de tabaco en alumnos de enseñanza secundaria de Cataluña. Rev. Atención Primaria, 45(6):315-323.Título artículo.

U.S. Department of Health and Human Services (1982). The health consecuences of smoking: Cancer. A report of the Surgeon General U.S. Department of Health and Human Services, Public Health Service, Office on Smoking and Health. DHHS Publication No. (PHS) 82-50179Título artículo.

U.S. Department of Health and Human Services (1988). The health consecuences of smoking: Nicotine addiction. A report of the Surgeon General U.S. Department of Health and Human Services, Public Health Service, Centers for Disease Control, Center for Health Promotion and Education, Office on Smoking and Health. DHHS Publication No. (CDC) 88-8406

U.S. Department of Health and Human Services (1989). Reducing the health consecuences of smoking: 25 years of progress. A report of the Surgeon General U.S. Department of Health and Human Services, Public Health Service, Centers for Disease Control, Center for Chronic Disease Prevention and Health Promotion, Office on Smoking and Health. DHHS Publication

U.S. Department of Health and Human Services (1990). The health benefits of smoking cessation. A report of the Surgeon General U.S. Department of Health and Human Services, Public Health Service, Centers for Disease Control, Center for Chronic Disease Prevention and Health Promotion Office on Smoking and Health. DHHS Publication No. (CDC) 90-8416.

U.S. Department of Health and Human Services (1994). Preventing tobacco use among young people. A report of the Surgeon General U.S. Department of Health and Human Services, Public Health Service, Centers for Disease Control and Prevention, National Center for Chronic Disease Prevention and Health Promotion, Office on Smoking and Health. 